\title{
Continuous
}

\section{non-invasive monitoring of evolving acute severe asthma during sleep}

\author{
Paul C Deegan, Walter T McNicholas
}

\begin{abstract}
A spontaneous acute severe asthmatic attack was monitored non-invasively in a 27 year old sleeping female asthmatic subject. As the attack evolved there was a switch from predominant abdominal breathing (associated with inspiratory indrawing of the rib cage) to gradually increasing rib cage excursion (associated with inspiratory paradox of the abdominal wall with respect to the rib cage). Abdominal paradox increased progressively until it appeared to fill the whole of the inspiratory period of the rib cage, at which point rapid oxygen desaturation developed.
\end{abstract}

(Thorax 1994;49:613-614)

There have been no reports of continuous monitoring of the evolution of acute severe asthma from a stable baseline level to a state of acute respiratory distress. Previous studies have monitored patients after arrival in the casualty department when the attack was established. ${ }^{1-3}$ By chance we were able to monitor the evolution of acute severe asthma continuously and non-invasively following stable baseline measurements. As the subject was asleep we could observe the physiological effects of the attack, free of conscious influences.

Department of Respiratory Medicine W T McNicholas

\section{Respiratory Sleep Laboratory} P C Deegan

University College and St Vincent's Hospital, Dublin 4, Ireland

Reprint requests to: Dr W T McNicholas.

Received 21 June 1993 Accepted for publication 22 July 1993

\section{Case report}

A 27 year old non-obese woman, presenting with nocturnal asthma, was commenced on sustained release theophylline in addition to her existing medication of inhaled salbutamol alone, as part of a controlled trial of nocturnal asthma treatment, with good effect.

After three weeks an overnight sleep study was performed in a dedicated sleep laboratory using standard polysomnography ${ }^{4}$ which included inductance plethysmography (Respitrace), electrocardiography, and ear oximetry. On arrival the patient reported a productive cough with mild dyspnoea and wheeze for three days, but not of sufficient severity to prompt her to seek treatment. She had no focal signs on examination and her $\mathrm{FEV}_{1}$ was $2.0 \mathrm{l}$ $(63 \%$ predicted). She felt well enough to undergo the study and received salbutamol $100 \mu \mathrm{g}$ by inhaler before lights out (00.15).

She slept well without waking despite significant falls in arterial oxygen saturation $\left(\% \mathrm{SaO}_{2}\right)$ and abnormal thoracoabdominal movements. When $\% \mathrm{SaO}_{2}$ fell to $80 \%$ at 05.15 she was woken up and the study terminated. On wakening she was very breathless with diffuse bronchospasm. FEV , had fallen to 0.71 (22\% predicted). Salbutamol $400 \mu$ g given by large spacer had no effect, but she responded to $5 \mathrm{mg}$ salbutamol and $500 \mu \mathrm{g}$ ipatropium bromide by nebuliser and her $\% \mathrm{SaO}_{2}$ rose to $96 \%$.

There was no clinical or radiological evidence of pneumonia or atelectasis. She subsequently made a rapid recovery on a tapering dose of oral prednisolone, oral erythromycin, and inhaled beclomethasone in addition to her existing medication. She had two further sleep studies at later dates, both of which were normal.

The principal breathing patterns identified during sleep are shown in fig 1 . A sequence was seen as the attack unfolded. A normal pattern was seen early in the study, with in phase movement of the rib cage, abdomen, and sum channels of the Respitrace (fig 1A). The onset of the acute attack is inferred by a

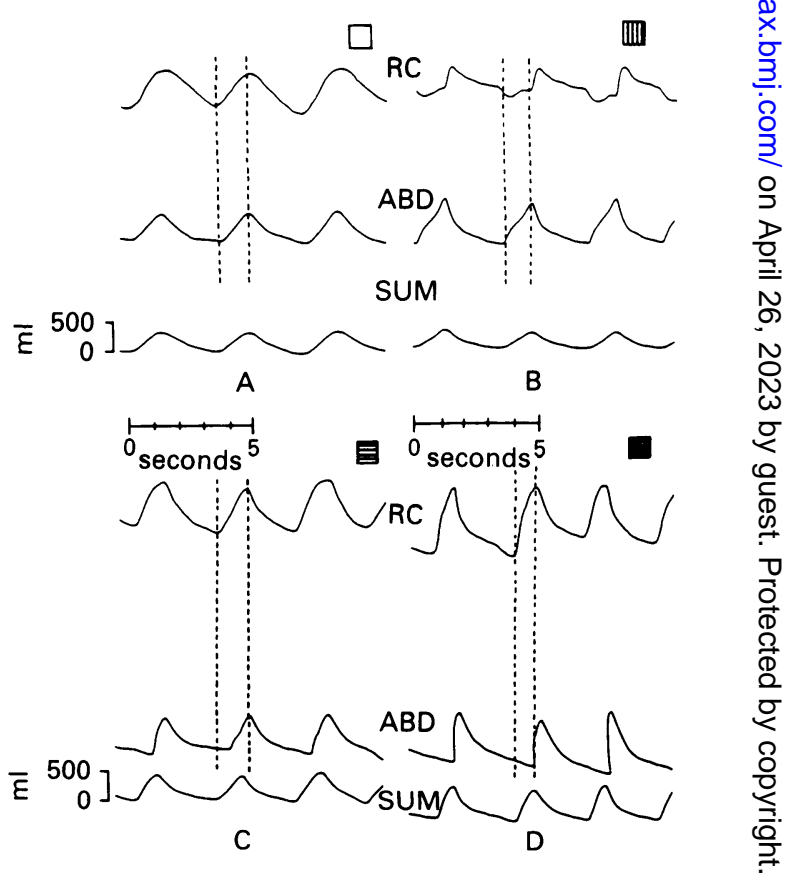

Figure 1 Principal Kespitrace patterns of

thoracoabdominal motion. ( $A$ ) normal pattern; (B) rib cage paradox; (C) partial inspiratory abdominal paradox; and (D) complete inspiratory paradox. $R C=$ rib cage channel; $A B D=$ abdominal channel; $S U M=$ sum channel. The inspiratory period is delineated by the two vertical dashed lines. The boxes are a key, referring to the principal pattern seen as the study progresses. 

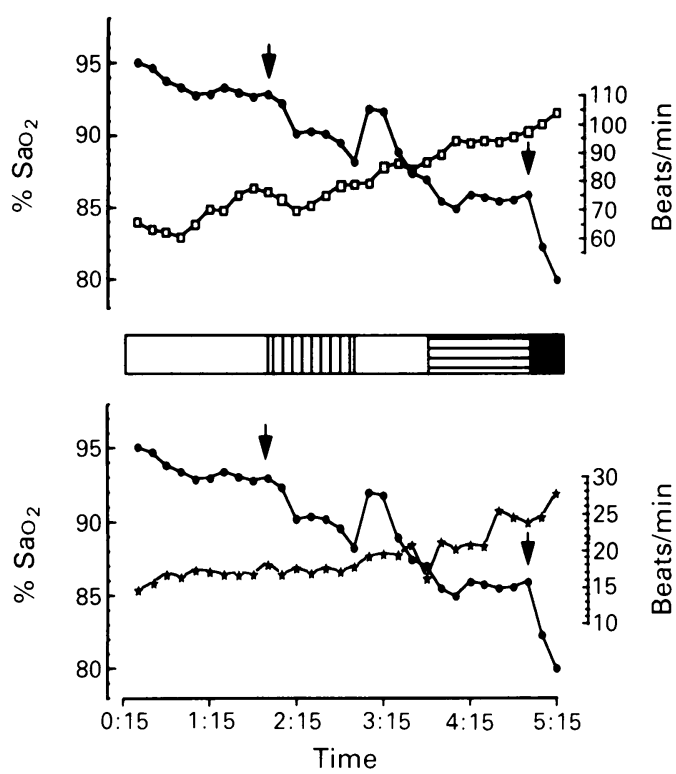

Figure 2 Relationship of heart rate (open squares, upper graph) and respiratory rate (stars, lower graph to oxygen saturation (closed circles), each expressed as mean values for successive 10 minute periods. The grid between the panels refers to patterns of abnormal

thoracoabdominal motion (see fig 1 for key). The arrows indicate onset of abrupt drops in $\% \mathrm{SaO}_{2}$ (see text).

marked drop in $\mathrm{SaO}_{2}$ (fig 2, left arrow) coincidental with the onset of inspiratory rib cage paradox (fig 1B) and a relative increase in amplitude of the abdomen channel, suggesting increased diaphragmatic activity as the cause of rib cage paradox. This pattern was abruptly terminated by the development of an augmented rib cage signal with a return to in phase rib cage and abdominal contractions and a transient rise in $\mathrm{SaO}_{2}$ (fig 2), falling again after 25 minutes as the attack proceeded.

This fall halted with the appearance of abdominal paradox during the early phase of inspiration (fig $1 \mathrm{C}$ ), with $\% \mathrm{SaO}_{2}$ remaining steady for about 45 minutes implying a beneficial effect. However, abdominal paradox became more marked as the attack progressed further, until complete paradox developed (fig 1D) and $\mathrm{SaO}_{2}$ fell rapidly over the space of 20 minutes until the patient was woken.

Heart rate rose progressively but only reached 100 beats/minute with a consistent fall in $\% \mathrm{SaO}_{2}$ to $86-87 \%$. On wakening the heart rate rose immediately from 106 to 123 beats/ minute. Respiratory rate did not change following wakening, but the breathing pattern became markedly irregular. Transient movement arousals were characterised by an increased sum, associated with a consistent increase in the rib cage component.

\section{Discussion}

This patient's clinical history and findings are typical of acute severe asthma. The findings during both of her follow up nights were comparable with other reports. ${ }^{5-7} \mathrm{Rib}$ cage paradox during REM sleep has been reported in asthmatic patients and is thought to represent the effects of diaphragmatic contraction on a rib cage made pliable by the normal loss of intercostal muscle tone during REM sleep. ${ }^{6}$ This patient differed in that rib cage paradox occurred during non-REM sleep, when intercostal muscle tone is relatively preserved. Abdominal paradox during non-REM sleep in asthmatic patients is thought to be a compensatory mechanism related to increased force of contraction of the rib cage,${ }^{6}$ and also appeared to benefit this subject. When abdominal paradox extended to the whole of inspiration, however, rapid oxygen desaturation occurred.

Failure of this subject to awaken fully, despite evidence of significant hypoxaemia and bronchoconstriction, is not altogether unusual. $^{89}$ The rate of transient arousals was 23 per hour just before wakening, which suggests that she was close to spontaneous wakening.

The rise in heart rate, usually regarded as a useful indicator of severity of asthma, ${ }^{10}$ was relatively small. Furthermore, the abrupt rise in heart rate when the patient was woken up suggests that part of the tachycardia seen in acute asthma is due to anxiety. The drop in $\% \mathrm{SaO}_{2}$ was not linear, with a transient rise after the ending of rib cage paradox and a levelling off initially with abdominal paradox. Individual arterial blood gas estimations during these periods, therefore, may not have accurately reflected the overall downward trend.

1 Hillman DR, Prentice L, Finucane KE. The pattern of breathing in acute severe asthma. Am Rev Respir Dis 1986;133:587-92.

2 Lavietes MH, Grocela JA, Maniatis T, Potulski F, Ritter A, Sunderam G. Inspiratory muscle strength in asthma. Chest 1988;93:1043-8.

3 Kesten S, Maleki-Yazdi MR, Sanders BR, Wells JA, McKillop SL, Chapman KR, et al. Respiratory rate during acute asthma. Chest 1990;97:58-62.

4 Rechtshaffen A, Kales A. A manual of standardised terminology, techniques and scoring system for sleep stages in nology, techniques and scoring system for sleep stages in
human subjects. Washington: National Institutes of human subjects. Washington:

5 Tabachnik E, Muller NL, Levison H, Bryan AC. Chest wall mechanics and pattern of breathing during sleep in asthmatic adolescents. Am Rev Respir Dis 1981;124:26973.

6 Issa FG, Sullivan CE. Respiratory muscle activity and thoracoabdominal motion during acute episodes of asthma during sleep. Am Rev Respir Dis 1985;132:9991004.

7 Deegan PC, McNicholas WT. Comparison of a sustainedrelease theophylline (Uniphyllin) with a long-acting inhaled beta-agonist (Salmeterol) in the management of nocturnal asthma. Am Rev Respir Dis 1993;147:A979.

nocturnal asthma. Am Rev Respir Dis 1993;147:A979.
Berthon-Jones M, Sullivan CE. Ventilatory and arousal Berthon-Jones $M$, Sullivan CE. Ventilatory and arousal
responses to hypoxia in sleeping humans. Am Rev Respir Dis 1982;125:632-9.

9 Gleeson K, Zwillich CW, White DP. The influence of increasing ventilatory effort on arousal from sleep. $\mathrm{Am}$ Rev Respir Dis 1990;142:295-300.

10 Fishl MA, Pitchenik A, Gardner LB. An index predicting relapse and need for hospitalisation in patients with acute bronchial asthma. $N$ Engl f Med 1981;305:783-9. 